\title{
Transient increases in serum $\alpha$ fetoprotein and protein induced by vitamin $K$ antagonist II levels following proton therapy does not necessarily indicate progression of hepatocellular carcinoma
}

\author{
MAIKO YOSHIDA $^{1-3}$, HIROYUKI OGINO ${ }^{1}$, HIROMITSU IWATA ${ }^{1}$, YUKIKO HATTORI ${ }^{1}$, \\ SHINGO HASHIMOTO ${ }^{1}$, KOICHIRO NAKAJIMA ${ }^{1}$, SHIGERU SASAKI ${ }^{4}$, MASAKI HARA ${ }^{4}$, \\ YOSHITAKA SEKIDO ${ }^{2,3}$, JUN-ETSU MIZOE ${ }^{1,5}$ and YUTA SHIBAMOTO ${ }^{6}$
}

\author{
${ }^{1}$ Department of Radiation Oncology, Nagoya Proton Therapy Center, Nagoya City West Medical Center, Nagoya, \\ Aichi 462-8508; ${ }^{2}$ Division of Cancer Biology, Aichi Cancer Center Research Institute, Nagoya, Aichi 464-8681; \\ ${ }^{3}$ Department of Molecular and Cellular Oncology, Field of Cancer Pathology and Informatics, Nagoya University Graduate \\ School of Medicine, Nagoya, Aichi 466-8550; ${ }^{4}$ Department of Diagnostic Radiology, Nagoya City West Medical Center, \\ Nagoya, Aichi 462-8508; ${ }^{5}$ Osaka Heavy Ion Therapy Center, Osaka 540-0008; ${ }^{6}$ Department of Radiology, \\ Nagoya City University Graduate School of Medical Sciences, Nagoya, Aichi 467-8601, Japan
}

Received August 14, 2018; Accepted December 31, 2018

DOI: $10.3892 / \mathrm{ol} .2019 .9922$

\begin{abstract}
Transient increases in $\alpha$-fetoprotein (AFP) and protein induced by vitamin $\mathrm{K}$ antagonist II (PIVKA-II), so-called flares, are frequently observed after treatment of hepatocellular carcinoma (HCC). In the present study, changes in AFP and PIVKA-II levels after proton therapy (PT), and the relationship between the flare phenomenon and clinical response were investigated. In 82 patients with stage I/II HCC (59 with no recurrence and 23 with out-of-field recurrence within 1 year), serum AFP and PIVKA-II levels were measured at 1, 3, 6, 9 and 12 months post-PT. AFP and PIVKA-II flares were defined as a $>20 \%$ increase from the preceding serum level above $20 \mathrm{ng} / \mathrm{ml}$ (AFP) or $40 \mathrm{mAU} / \mathrm{ml}$ (PIVKA-II), followed by a $>20 \%$ drop. Among the 59 patients with no recurrence, $3(5.1 \%)$ had an AFP flare, while $23(39 \%)$ had a PIVKA-II flare. The median time to AFP and PIVKA-II flare peaks was 1 and 6 months, respectively. In 4 patients, PIVKA-II flares were observed twice during follow-up. In 1 patient, AFP and PIVKA-II flares were observed simultaneously at 1 month post-PT. The PIVKA-II level pre-PT was significantly higher in the PIVKA-II flare-positive group compared with that in the flare-negative group $(\mathrm{P}=0.015$, odds ratio $4.3,95 \%$ confidence
\end{abstract}

Correspondence to: Professor Yuta Shibamoto, Department of Radiology, Nagoya City University Graduate School of Medical Sciences, 1 Kawasumi, Mizuho-cho, Mizuho-ku, Nagoya, Aichi 467-8601, Japan

E-mail: yshiba@med.nagoya-cu.ac.jp

Key words: proton therapy, hepatocellular carcinoma, $\alpha$-fetoprotein, protein induced by vitamin $\mathrm{K}$ absence or antagonist-II, flare phenomena interval, 1.3-14.0). In the 23 patients with out-of-field recurrence, the median increase rate of PIVKA-II (203\%) was higher than that in the PIVKA-II-flare-positive group (111\%, $\mathrm{P}=0.035$ ) and the time to recurrence (median, 9 months) was longer than the time to peak AFP level (1 month) in the AFP-flare-positive group ( $\mathrm{P}=0.033)$. There was no significant association between flares and clinical response. Increases in AFP and PIVKA-II levels following PT should be assessed with caution to avoid misinterpretation of therapeutic outcome.

\section{Introduction}

Hepatocellular carcinoma (HCC) is the fifth most common tumor and the second most common cause of cancer-related death worldwide (1). In the last decades, with marked improvements in medical technology and well-established screening programs for high-risk patients, the prognosis of HCC patients has improved (2). However, although 5-year survival rates after curative treatments like hepatectomy, radiofrequency ablation (RFA), and liver transplantation are as high as $50-70 \%$, only $20-40 \%$ patients were able to receive these therapies due to their comorbidities and/or tolerability, in addition to late diagnosis $(2,3)$. Recently, proton therapy (PT) has offered promising results to overcome these limitations $(4,5)$. To test whether PT is non-inferior to hepatectomy for resectable HCC, a non-randomized controlled study of PT vs. hepatectomy for resectable HCC started in June 2017 (Japan Clinical Oncology Group, JCOG1315C, SPRING study).

After the primary treatment of $\mathrm{HCC}$, active surveillance is essential because the 5-year tumor recurrence rate remains as high as over $50 \%$ after curative treatment, including PT (5-8). In order to enhance the sensitivity and specificity for HCC detection, measurement of serum tumor markers, especially alpha-fetoprotein (AFP) and protein induced by vitamin $\mathrm{K}$ antagonist II (PIVKA-II, also known as des-gamma-carboxy 
prothrombin), is recommended to complement imaging examination and is used as a predictor of progression of HCC $(9,10)$. Increases in these biomarker levels during follow-up after primary treatments are generally considered to indicate a failure of treatment and tumor progression. In actual clinical settings, however, transient increases in the biomarkers are frequently observed despite the clinical response. This phenomenon is called flare or surge, and may lead to misinterpretation of tumor progression.

Several investigators have reported transient increases in several serum tumor markers during the first few weeks after initiation of chemotherapy in different cancers. Some researchers correlated the flare phenomena with better prognosis (11-13). However, to the best of our knowledge, no study has yet focused on the transient increase in serum tumor markers during a longer follow-up period after local therapy. In addition, transient enlargement of HCC on diagnostic imaging is also observed during follow-up, but this phenomenon is poorly understood. In this study, therefore, we investigated changes in serum AFP and PIVKA-II levels and tumor size on contrast-enhanced magnetic resonance imaging (MRI) during a 1-year follow-up period after PT, and analyzed factors potentially associated with this flare phenomenon.

\section{Materials and methods}

Patients. Between September 2013 and March 2016, 97 patients with stage I/II HCC (TNM Classification of Malignant Tumours Ver. 7, the Union for International Cancer Control) were prospectively treated with PT according to the protocols approved by the institutional review board [no. 12-02-22 (18)]. Among them, one patient who were taking warfarin and 14 patients who were lost to the regular follow-up (blood tests and MRI could not be performed as scheduled) were excluded from analysis. In addition, since it is difficult to distinguish the transient increase in the tumor markers caused by PT from the elevation caused by recurrence outside of the PT field, 23 patients who had an out-of-field recurrence within 12 months after PT were analyzed separately for comparison. Thus, 59 HCC patients were analyzed with respect to the flare phenomena in this study (Fig. 1). Table I shows patient and treatment characteristics. Sixteen $(27 \%)$ of the 59 patients had received previous treatments such as hepatectomy, RFA, transarterial embolization, and transarterial chemoembolization.

Evaluation of tumor markers. Serum levels of AFP and PIVKA-II were measured before PT, and at 1, 3, 6, 9, and 12 months after PT. The cut-off levels for serum AFP and PIVKA-II were $20 \mathrm{ng} / \mathrm{ml}$ or $40 \mathrm{mAU} / \mathrm{ml}$, respectively, at our institution. AFP and PIVKA-II flares were defined as a $>20 \%$ increase from the level at the preceding measurement in the range above the cut-off level, followed by a $>20 \%$ drop upon subsequent measurements, according to the criteria used in studies of the flare phenomenon after chemotherapy $(11,12)$. If serum elevation was observed at 12 months after PT, a drop in the serum level was confirmed thereafter. Although consecutive rises were counted as a single flare, inconsecutive increases were analyzed independently. For comparison, serum elevations in AFP and PIVKA-II at the time of recurrence were also defined by the same criteria $(>20 \%$ increase and over cut-off levels). AFP and PIVKA-II were measured by chemiluminescence immunoassay (CLIA).

Evaluation of contrast-enhanced magnetic resonance imaging. Gd-EOB-DTPA-enhanced dynamic MRI was performed with $2.5 \mathrm{~mm}$ slice thickness, and the longest diameter of the lesion was measured on the hepatobiliary phase images. Even when a hypointensity nodule was observed on T1and/or T2-weighted images, disappearance of the target lesion in the hepatobiliary phase was regarded as a complete response (CR). When the tumor diameter was difficult to measure, arterial, portal venous, and diffusion-weighted images were also evaluated. The tumor diameter was measured by two radiologists who had access to the patients' clinical information. The inter-observer differences were less than $7 \%$ in all cases, and the two measured diameters were averaged. Transient enlargement was defined as a $>10 \%$ increase in the longest tumor diameter between consecutive MR imaging studies, followed by a $>10 \%$ decrease upon subsequent measurements. Tumor progression was diagnosed by consecutive enlargement at 2 follow-up examinations over 6 months. Responses to treatment were evaluated with dynamic MRI studies following the RECIST guidelines (14).

Proton therapy. PT was performed with PROBEAT-III (Hitachi Ltd., Tokyo, Japan) at Nagoya Proton Therapy Center. Details of the system have been described previously $(15,16)$. Our methods of PT planning and delivery have been described in detail previously $(17,18)$. Prior to PT, patients underwent gold marker implantation near or inside the tumor for target matching and replanning. 2-mm-thick computed tomography (CT) images at the expiration phase were taken and fused with Gd-EOB-DTPA-enhanced dynamic MRI or contrast-enhanced CT, which were used for gross tumor volume (GTV) contouring. In addition to the GTV, the internal gross target volume included respiratory motions, and 3-6 mm margins were added as an intra/interfractional margin for the internal clinical target volume (ICTV). The planning target volume (PTV) included ICTV and setup margins. The delivered dose of PT was $66 \mathrm{GyE}$ in 10 fractions for peripheral HCC located more than $2 \mathrm{~cm}$ from the porta hepatis and $72.6 \mathrm{GyE}$ in 22 fractions for HCC located within $2 \mathrm{~cm}$ of the porta hepatis. The ideal PTV dose constraint was D98\% (dose received by $98 \%$ of the volume of the PTV) $>95 \%$, which was adjusted to satisfy the organs at risk (OARs) dose limit of the liver-GTV and intestinal tract. The planning constraint for the standard liver volume (remnant liver volume irradiated $<1 \mathrm{GyE}$ ) was $\geq 35 \%$.

Statistical analysis. Factors associated with the PIVKA-II flare phenomena were analyzed by univariate analysis using the Fisher's exact test as well as multivariate analyses using logistic regression model. Age, gender, etiology, Child Pugh class, stage, previous treatment, protocol, tumor marker rise before PT, transient increase in AFP and size, and response at 12 months were included as variables for univariate analysis. Variables with a P-value less than 0.1 in the univariate analysis (non $\mathrm{HBV} / \mathrm{HCV}$ disease and PIVKA-II rise before PT) were included in the multivariate analysis. Relationship between the PIVKA-II or AFP flare and out-of-field recurrence was also assessed by Fisher's exact test. Mann-Whitney U test was used 
Table I. Patient characteristics.

\begin{tabular}{|c|c|c|c|c|c|}
\hline \multirow[b]{2}{*}{ Variable } & \multicolumn{4}{|c|}{ No recurrence } & \multirow[b]{2}{*}{$\begin{array}{l}\text { Out-of-field } \\
\text { recurrence }\end{array}$} \\
\hline & Total & AFP flare & PIVKA-II flare & $\begin{array}{c}\text { Transient } \\
\text { increase on MRI }\end{array}$ & \\
\hline Number of patients & 59 & 3 & 23 & 3 & 23 \\
\hline \multicolumn{6}{|l|}{ Age (years) } \\
\hline Median (range) & $67(38-85)$ & $66(63-74)$ & $67(38-85)$ & $68(65-81)$ & $72(40-83)$ \\
\hline \multicolumn{6}{|l|}{ Sex } \\
\hline Male & $38(64)$ & $1(33)$ & $13(57)$ & $2(67)$ & $16(70)$ \\
\hline Female & $21(36)$ & $2(67)$ & $10(43)$ & $1(33)$ & $7(30)$ \\
\hline \multicolumn{6}{|l|}{ Etiology } \\
\hline HCV infected & $27(46)$ & $2(67)$ & $10(43)$ & $1(33)$ & $11(48)$ \\
\hline HBV infected & $15(25)$ & $0(0)$ & $3(13)$ & $1(33)$ & $5(22)$ \\
\hline Non HBV, non HCV & $17(29)$ & $1(33)$ & $10(43)$ & $1(33)$ & $7(30)$ \\
\hline \multicolumn{6}{|l|}{ Child pugh class } \\
\hline A & $51(86)$ & $2(67)$ & $21(91)$ & $3(100)$ & $16(70)$ \\
\hline $\mathrm{B}$ & $8(14)$ & $1(33)$ & $2(9)$ & $0(0)$ & $7(30)$ \\
\hline \multicolumn{6}{|l|}{ Stage, n (\%) } \\
\hline I & $49(83)$ & $3(100)$ & $18(78)$ & $3(100)$ & $7(30)$ \\
\hline II & $10(17)$ & $0(0)$ & $5(22)$ & $0(0)$ & $16(70)$ \\
\hline \multicolumn{6}{|l|}{ Previous treatment } \\
\hline Yes & $16(27)$ & $0(0)$ & $4(17)$ & $1(33)$ & $12(52)$ \\
\hline No & $43(73)$ & $3(100)$ & $19(83)$ & $2(67)$ & $11(48)$ \\
\hline \multicolumn{6}{|l|}{ Protocol (GyE/fractions) } \\
\hline $66 / 10$ & $46(78)$ & $2(67)$ & $18(78)$ & $2(67)$ & $15(65)$ \\
\hline $72.6 / 22$ & $13(22)$ & $1(33)$ & $5(22)$ & $1(33)$ & $8(35)$ \\
\hline \multicolumn{6}{|c|}{ Tumor marker rise before PT } \\
\hline AFP & $23(39)$ & $3(100)$ & $8(35)$ & $0(0)$ & $11(48)$ \\
\hline PIVKA-II & $27(46)$ & $1(33)$ & $16(70)$ & $1(33)$ & $14(61)$ \\
\hline \multicolumn{6}{|l|}{ Response at 12 months } \\
\hline $\mathrm{CR}$ & $33(56)$ & $2(67)$ & $10(43)$ & $0(0)$ & $2(9)$ \\
\hline PR & $17(29)$ & $1(33)$ & $8(35)$ & $1(33)$ & $20(87)$ \\
\hline SD & $5(8)$ & $0(0)$ & $4(17)$ & $2(67)$ & $1(4)$ \\
\hline
\end{tabular}

Except for age, numbers of patients and percentages in each group in parentheses are shown; CR, complete response; PR, partial response; $\mathrm{SD}$, stable disease; PT, proton therapy.

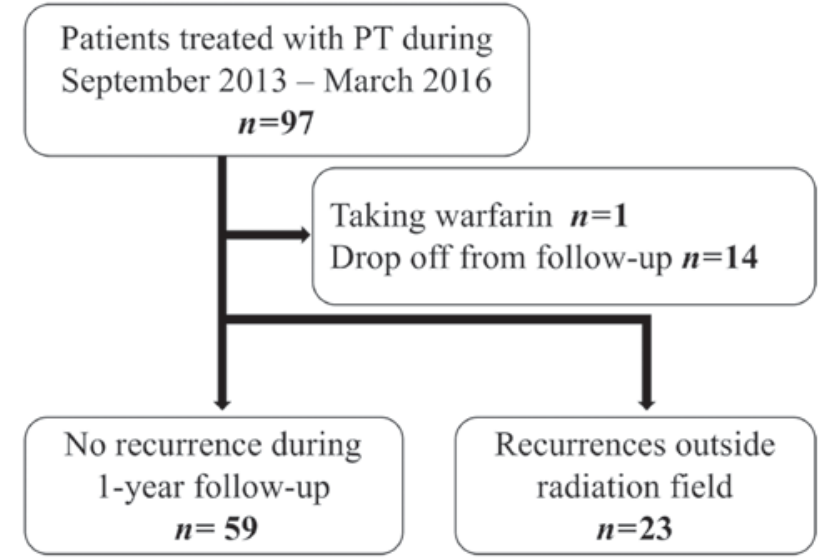

Figure 1. Patient selection flow chart. PT, proton therapy. to compare the median increase rates of PIVKA-II and AFP, and times to peak PIVKA-II/AFP levels, times to recurrence between the flare-positive and out-of-field recurrence groups, pretreatment levels of PIVKA-II between the groups with and without flare phenomena, and tumor size, GTV, and PTV with and without the flare. A P-value $<0.05$ was considered significant. Statistical analyses were performed using EZR (Easy R, Saitama Medical Center, Jichi Medical University, Saitama, Japan), which is a modified version of $\mathrm{R}$ commander (version 1.6-3) (19).

\section{Results}

Pretreatment levels of AFP and PIVKA-II were elevated in 39 and $46 \%$, respectively, of the patients with no recurrence and, 48 and 
$61 \%$, respectively, of the patients with out-of-field recurrence (Table I). Among the 59 patients with no recurrence, 23 (39\%) had a PIVKA-II flare and the median time to the flare peak was 6 months (Table II). The median transient increase rate compared with the level at preceding examinations (i. e., level after elevation minus level before elevation/level before elevation) was $111 \%$ (range, 24-3740\%) for PIVKA-II. On the other hand, AFP flares were observed in $5.1 \%$. The median time to the flare was 1 month and the median rate of increase was $32 \%$ (range, 30-42\%).

No patient developed in-field recurrence during the 12-month follow-up, whereas 23 patients in the other group developed out-of-field recurrence (Fig. 1). Table III compares data on AFP and PIVKA-II levels between the groups with or without recurrence. In the 23 patients with out-of-field recurrence, the median increase rate of PIVKA-II (203\%, range 70-2773) was higher than that in the PIVKA-II-flare-positive group $(111 \%$, range $24-3740, \mathrm{P}=0.035)$. The median time to recurrence was 9 months (range, 1-12 months) and it was longer than the time to peak AFP level (1 month) in the AFP-flare-positive group $(\mathrm{P}=0.033)$. Two of $12(17 \%)$ patients with no pretreatment AFP elevation showed AFP elevation at recurrence, and four of $9(44 \%)$ patients with no pretreatment PIVKA-II elevation showed PIVKA-II elevation at recurrence. Seven of 11 (64\%) patients with pretreatment AFP elevation showed AFP elevation at recurrence, and 11 of 14 (79\%) patients with pretreatment PIVKA-II elevation showed PIVKA-II elevation at recurrence.

In the no-recurrence group, the median longest tumor diameter was $22 \mathrm{~mm}$ (range $8-133 \mathrm{~mm}$ ) at diagnosis. Three $(5.1 \%)$ exhibited transient enlargement. The median time to peak enlargement was 1 month (range, 1-3 months) after PT, and the median increase rate was $22 \%$ (range, 14-30\%) (Table II). Table IV summarizes tumor size, GTV, and irradiated liver volume (PTV) in groups with or without the flare phenomena. Median GTV of tumors with AFP flare tended to be greater than that without AFP flare $(\mathrm{P}=0.094)$. Fig. 2A shows a representative case of pseudoprogression in which the tumor size enlarged at 1 month after PT but shrinked thereafter. Although their sizes increased, contrast enhancement in the arterial phase was weaker than the pretreatment levels. For reference, Fig. 2B shows a case with in-field recurrence developing after the 12-month follow-up. The tumor size increased at 15 months after PT and further at 18 months. This patient had a PIVKA-II flare at 1 month after PT.

AFP and PIVKA-II flares were observed during two or more follow-up visits in one and 8 patients, respectively. In one patient, the transient increase of PIVKA-II lasted from 3 to 9 months, which was the longest flare duration. PIVKA-II flares were also observed twice during follow-up in four patients (Fig. 3). Both PIVKA-II flare and transient tumor enlargement on MRI were observed in 2 patients (Fig. 4). Among them, 1 patient also had simultaneous PIVKA-II flare and transient enlargement at 1 month after PT (Fig. 4B).

Table $\mathrm{V}$ shows the univariate and multivariate analyses used to detect factors associated with the flare phenomenon of PIVKA-II. Age ( $<$ or $\geq$ median, 67 years), gender, infection status of $\mathrm{HCV}$ and $\mathrm{HBV}$, Child-Pugh class, stage, previous treatment, PT protocol, tumor marker elevation before PT, transient increase in AFP or size, and response at 12 months after PT were selected as variables. In the multivariate analysis, pretreatment elevation of PIVKA-II before PT was

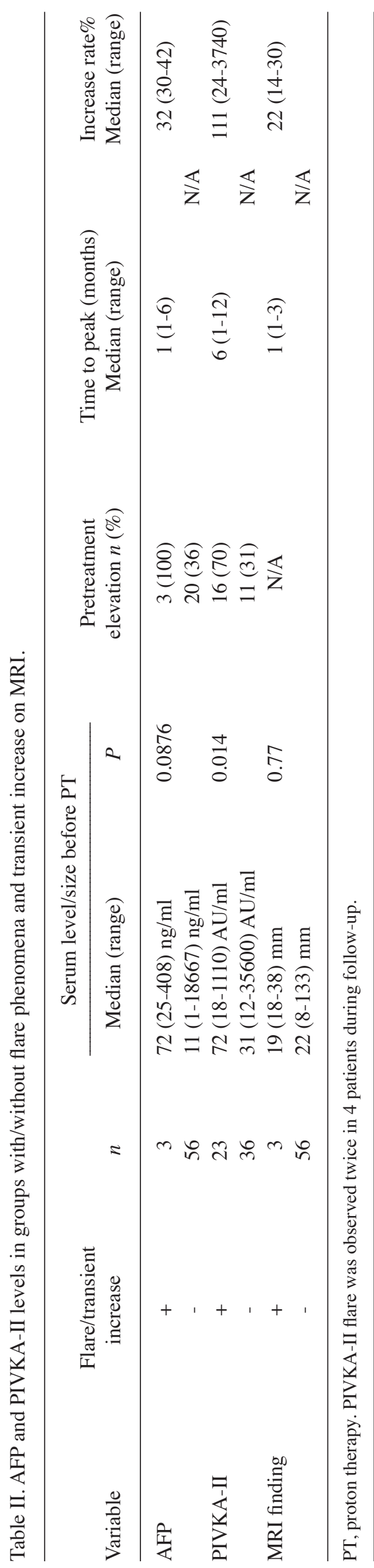


associated with PIVKA-II flares ( $\mathrm{P}=0.015$, odds ratio 4.3, 95\% confidence interval 1.3-14.0). Fig. 5 shows pretreatment PIVKA-II serum levels in no-recurrence groups with or without PIVKA-II flares; the PIVKA-II levels were higher in the group with the flare than in the group without it (median, 72 and $30.5 \mathrm{mAU} / \mathrm{ml}$, respectively; $\mathrm{P}=0.014)$.

Regarding the relationship between the flare phenomena and out-of-field recurrence, PIVKA-II flare tended to be more often observed in the no-recurrence group $(23 / 59=39 \%)$ than in the out-of-field recurrence group $(4 / 23=17 \% ; \mathrm{P}=0.072)$, but AFP flare was not related to out-of-field recurrence $(\mathrm{P}=0.34)$.

\section{Discussion}

Besides imaging studies, serum levels of AFP and PIVKA-II are good surveillance tools due to their wide utility, and they complement image examinations to maximize early detection of HCC (20). Recent studies suggested that the combination of AFP and PIVKA-II improved the detection rate of HCC, and was useful to measure treatment response and monitor recurrence $(21,22)$. As the combination of PIVKA-II and AFP may increase sensitivity without decreasing specificity, the guidelines of the Japan Society of Hepatology recommended to measure these biomarkers during HCC surveillance (10). A recent study showed that the tumor markers might elevate at $0.5-1.5$ years before the HCC lesion becomes obvious on diagnostic imaging (23). Furthermore, AFP and PIVKA-II elevation may suggest micrometastases, which may result in early recurrence within weeks or months after primary treatment $(21,24)$. Therefore, elevation of tumor markers within 1 year after primary treatment requires special attention.

However, the present study indicated that transient increases could be observed in both AFP and PIVKA-II levels during the 12-month follow-up period after PT regardless of therapeutic response. Compared to the out-of-field recurrence, the PIVKA-II increase rates were considerably lower in the PIVKA-II-flare group ( $\mathrm{P}=0.035)$. The AFP-flare peak was observed earlier than in the recurrence group $(\mathrm{P}=0.033)$, but this was not the case for PIVKA-II $(\mathrm{P}=0.34)$. One case showed the transient increase lasting for more than 6 months and in other cases, the flare phenomena were observed twice during 12 months after PT. These observations might imply that several mechanisms could be involved in the transient increases. The flare phenomenon observed at early periods may be related to tumor cell lysis, as reported for several cancers treated by chemotherapy (11). In the AFP-flare group, the tumor size (GTV) tended to be larger and the flare tended to be observed at early periods; this observation would support the hypothesis that the early flares would be caused by HCC lysis. On the other hand, AFP elevation was also reported to be triggered by acute hepatitis (25), and it was thought that damages to liver cells may result in activation of hepatocyte turnover and elevation of these tumor markers (26). In contrast, the late flare may be due in part to focal liver parenchymal damage and/or compensatory enlargement of unirradiated liver caused by PT $(27,28)$. PIVKA-II transient increases were observed through a year period after PT and it was reported that liver cell damage triggered cell regeneration followed by an increase of the PIVKA-II serum level (23). Since AFP flare was not observed in the later period, the AFP flare in this study 
Table IV. Longest diameter, gross tumor volume (GTV), and planning target volume (PTV) in groups with or without flare phenomena.

\begin{tabular}{|c|c|c|c|c|c|c|}
\hline \multirow[b]{2}{*}{ Variable } & \multicolumn{3}{|c|}{ AFP } & \multicolumn{3}{|c|}{ PIVKA-II } \\
\hline & Flare $+(n=3)$ & Flare $-(n=56)$ & $P$ & Flare $+(n=23)$ & Flare $-(n=36)$ & $P$ \\
\hline $\begin{array}{l}\text { Longest diameter, } \mathrm{mm} \\
\text { Median (range) }\end{array}$ & $33(21-36)$ & $22(8-133)$ & 0.23 & $22(8-109)$ & $22(8-133)$ & 0.41 \\
\hline $\begin{array}{l}\mathrm{GTV}, \mathrm{cm}^{3} \\
\text { Median (range) }\end{array}$ & $30(17-36)$ & $10(2-1824)$ & 0.09 & $12(2-515)$ & $9(2-1824)$ & 0.26 \\
\hline $\begin{array}{l}\text { PTV }, \mathrm{cm}^{3} \\
\text { Median (range) }\end{array}$ & $93(80-96)$ & 49 (17-2359) & 0.17 & $51(18-855)$ & $50(17-2359)$ & 0.52 \\
\hline
\end{tabular}

A

Pseudoprogression

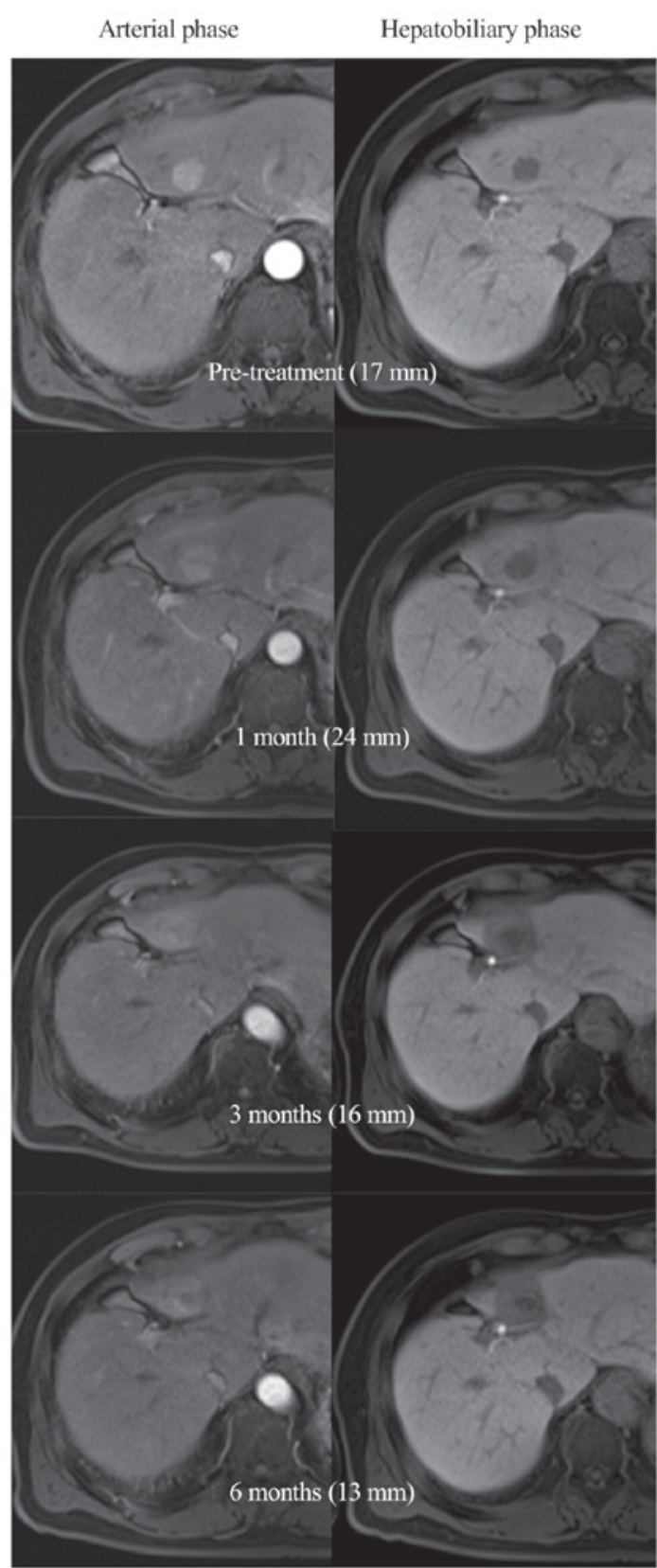

B

Progression

Arterial phase Hepatobiliary phase

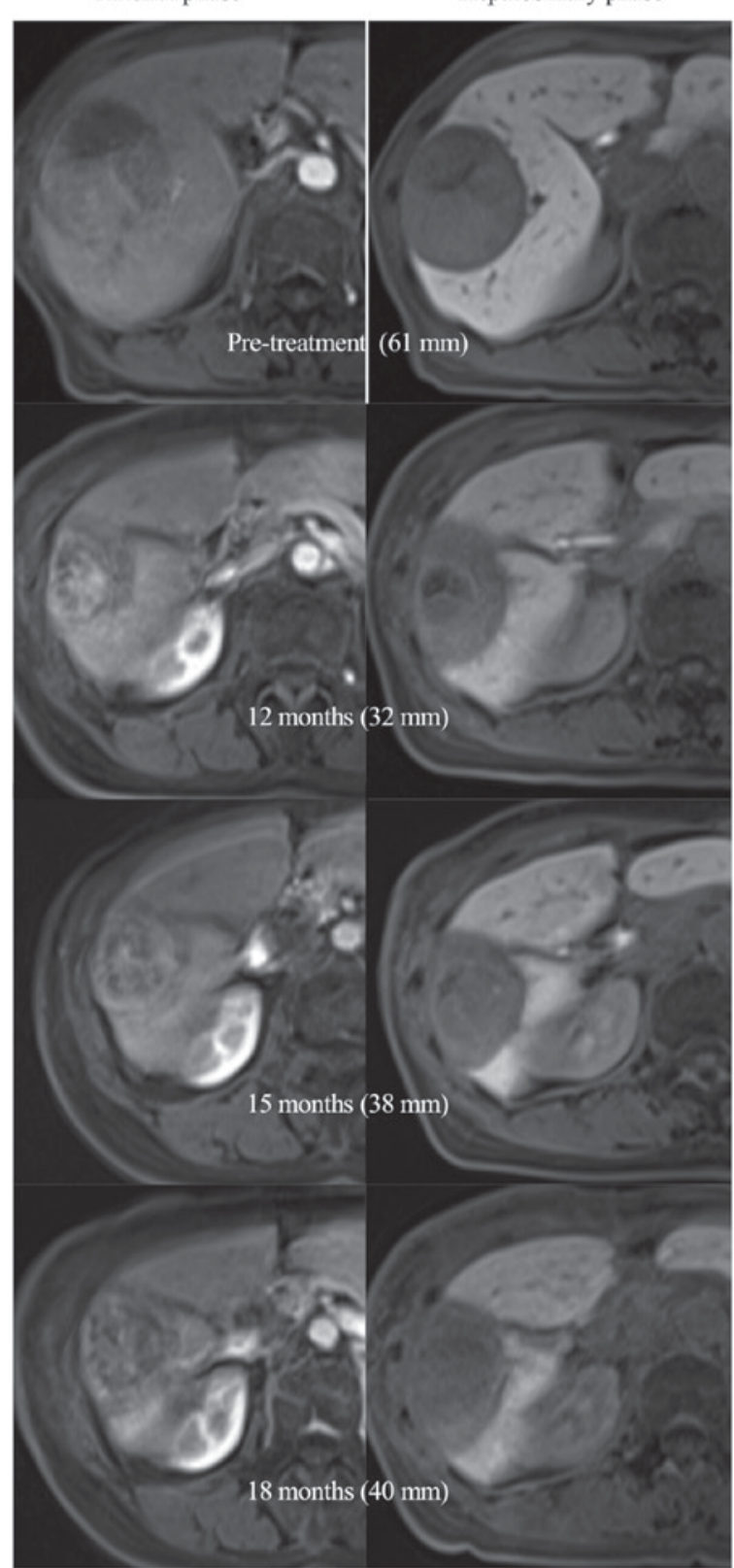

Figure 2. Contrast-enhanced T1-weighted images of representative cases of (A) pseudoprogression and (B) true progression. Longest diameters are shown in parentheses. (A) The tumor shadow was enlarged at 1 month post-PT and had shrunk at 3 months. (B) The tumor size at 1 year post-PT was smaller than pre-PT. However, the lesion enlarged at 15 months and continually sized up at 18 months post-PT. PT, proton therapy. 


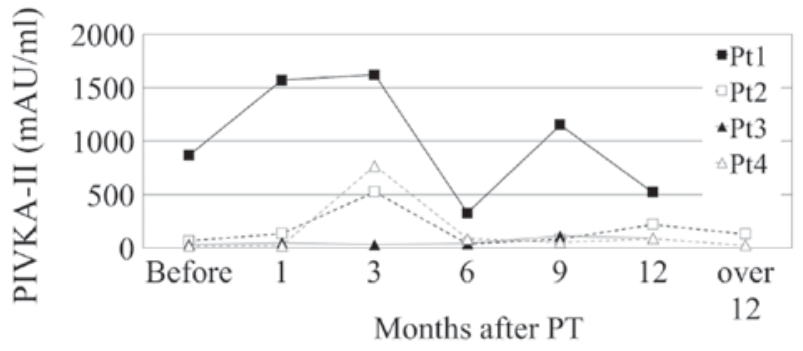

Figure 3. Transient increase observed twice during 12-month follow-up in 4 patients (Pt 1-4). x-axis indicates months after PT while $y$-axis shows serum levels of PIVKA-II (mAU/ml). The flare peak was observed at 3 and 9 months in Pt 1, at 3 and 12 months in Pt 2 and 4, and at 1 and 9 months in Pt 3. PT, proton therapy; Pt, patient; PIVKA-II, protein induced by vitamin $\mathrm{K}$ antagonist II.

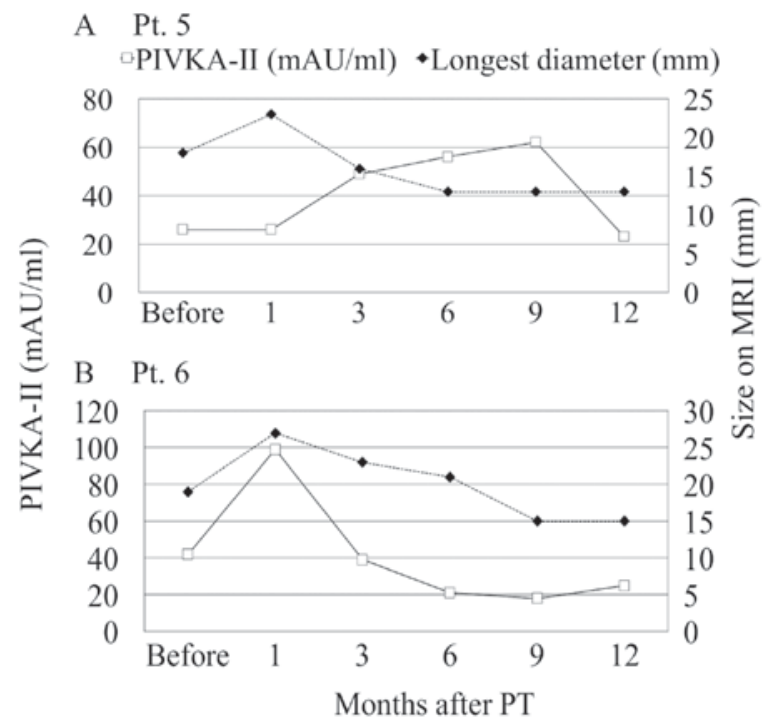

Figure 4. PIVKA-II flare and pseudoprogression on MRI observed in 2 patients, (A) Pt 5 and (B) Pt 6. x-axis indicates months after PT. y-axis (left) shows serum levels of PIVKA-II (mAU/ml) and y-axis (right) shows tumor size on MRI. MRI, magnetic resonance imaging; PT, proton therapy; $\mathrm{Pt}$, patient; PIVKA-II, protein induced by vitamin K antagonist II.

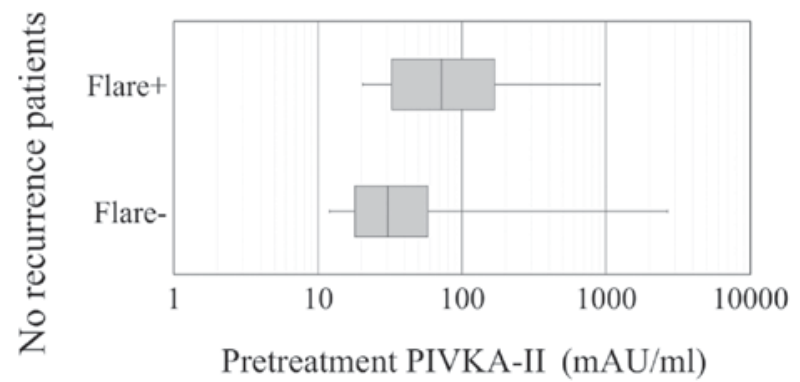

Figure 5. Box plot for PIVKA-II serum levels before PT in the PIVKA-II flare-positive and -negative groups with no recurrence $(n=59)$. The box plot indicates the lower quartile and upper quartile, and the middle line indicates the median serum level. Solid lines extended from the boxes indicate 95th and 5 th percentile of the data. PIVKA-II, protein induced by vitamin $\mathrm{K}$ antagonist II; PT, proton therapy.

might have been mainly triggered by HCC lysis. Therefore, increases of AFP level in the later period after PT may require careful attention to recurrences.
Table V. Univariate and multivariate analyses of factors associated with flare phenomena of PIVKA-II.

\begin{tabular}{lcc}
\hline & \multicolumn{2}{c}{$P$} \\
\cline { 2 - 3 } Variables & $\begin{array}{c}\text { Univariate } \\
\text { analysis }\end{array}$ & $\begin{array}{c}\text { Multivariate } \\
\text { analysis }\end{array}$ \\
\hline Age < vs. $\geq 67$ years & 1 & \\
Gender male vs. female & 0.41 & \\
Etiology & & \\
HCV infected & 0.8 & \\
HBV infected & 0.13 & \\
Non HBV, non HCV & 0.08 & \\
Child pugh class A vs. B & 0.46 & \\
Stage I vs. II & 0.49 & \\
Previous treatment & 0.24 & \\
Protocol 66 vs. 72.6 GyE & 1 & \\
Tumor marker rise before PT & & \\
AFP & 0.79 & \\
PIVKA-II & 0.0068 & \\
Transient increase & & \\
AFP & 0.55 & \\
Size on MRI & 0.55 & \\
Response at 12 M, CR vs. other & 0.28 & \\
\hline
\end{tabular}

In univariate and multivariate analyses, only pretreatment elevation of PIVKA-II before PT was associated with the PIVKA-II flare. It is not surprising that the flare phenomenon more often occurs in patients with high pretreatment tumor marker levels. In patients with a high pretreatment level, the flare phenomenon was seen in $59 \%$ (16/27). Even in patients with a normal pretreatment level, the phenomenon was observed in $22 \%$ (7/32). On the other hand, PIVKA-II elevation at out-of-field recurrence was observed in $79 \%$ (11/14) of patients with pretreatment PIVKA-II elevation and in $44 \%$ (4/9) of patients with no pretreatment elevation. Therefore, it seems difficult to estimate whether post-PT elevation of PIVKA-II is the flare or recurrence from the pretreatment level alone. Serial examinations are necessary to distinguish between the two, but the increase rate would be of some help, since the rate was higher in the recurrence group. In addition, simultaneous evaluation of AFP levels should also be helpful.

In our study, $5.1 \%$ of the patients exhibited transient enlargement of the lesion on MRI at 1-3 months after PT. Overall, contrast enhancement in the arterial phase was weaker than that of the pretreatment lesion, which implies tumor necrosis and decrease of HCC activity (29). Although the mechanism of the transient enlargement was unclear, a previous study indicated that radiation therapy could induce sinusoidal congestion and hyperemia in the early phase after irradiation (30). In that study, the target lesion in some patients also showed transient enlargement after radiation. PT would induce the same mechanism, which may result in pseudoprogression of the target lesion. Moreover, as shown in the present study, it is 
noteworthy that a transient elevation in the PIVKA-II level and pseudoprogression on MRI could be observed simultaneously in the early period after PT. In order to predict whether tumor recurrence will indeed occur, a dynamic imaging study should be performed to assess obvious arterial uptake and wash-out in the delayed phase as well as treatment-induced necrosis, which was recently proposed in modified RECIST and RECICLE to assess treatment response of $\mathrm{HCC}(31,32)$.

Our study has several limitations. The study size was relatively small and the patients had various backgrounds. In addition, the definition of flare in our study was arbitrary. Therefore, the conclusion might be biased by these factors. Moreover, as the follow-up duration in this study was relatively short, it was inconclusive whether the transient increase was related with eventual development of HCC. Further studies are required to ensure whether other locoregional treatments, such as RFA, also cause transient increases in tumor markers to investigate the mechanism of the flare phenomenon.

In summary, increases in tumor marker serum levels as well as tumor size on imaging examination should be assessed with extra caution to avoid misinterpretation of therapeutic outcome. The combination of the serum biomarkers AFP and PIVKA-II and dynamic imaging examination is essential to monitor tumor progression after PT.

\section{Acknowledgements}

The authors would like to thank Dr Kyoji Senoo and Dr Yoshiyuki Kuwahara at the Nagoya Proton Therapy Center for their help in this research.

\section{Funding}

This study was supported by Grants-in-Aid for JSPS KAKENHI (grant nos. 15K10005, 15K10006 and 16K10400).

\section{Availability of data and materials}

The datasets used and/or analyzed during the current study are available from the corresponding author on reasonable request.

\section{Authors' contributions}

MY, HO, YuS and JM designed the study. MY, HO, HI, YH, SH, KN, SS, MH, YuS, YoS and JM analyzed and interpreted the patient data regarding flare phenomena in tumor markers of HCC following proton therapy. YuS drafted the manuscript and revised it critically for important intellectual content. All authors read and approved the final manuscript.

\section{Ethics approval and consent to participate}

The protocols for this research were approved by the institutional review board [no. 12-02-22 (18)]. Written informed consents to participate in the present research were provided prior to PT.

\section{Patient consent for publication}

Written informed consents for the publication were obtained from all patients in this research.

\section{Competing interests}

The authors declare that they have no competing interests.

\section{References}

1. Ferlay J, Soerjomataram I, Dikshit R, Eser S, Mathers C, Rebelo M, Parkin DM, Forman D and Bray F: Cancer incidence and mortality worldwide: Sources, methods and major patterns in GLOBOCAN 2012. Int J Cancer 136: E359-E386, 2015.

2. Osaki Y and Nishikawa H: Treatment for hepatocellular carcinoma in Japan over the last three decades: Our experience and published work review. Hepatol Res 45: 59-74, 2015.

3. Llovet JM, Burroughs A and Bruix J: Hepatocellular carcinoma. Lancet 362: 1907-1917, 2003.

4. Sakurai H, Ishikawa $\mathrm{H}$ and Okumura T: Proton beam therapy in Japan: Current and future status. Jpn J Clin Oncol 46: 885-892, 2016.

5. Fukuda K, Okumura T, Abei M, Fukumitsu N, Ishige K, Mizumoto M, Hasegawa N, Numajiri H, Ohnishi K, Ishikawa $\mathrm{H}$, et al: Long-term outcomes of proton beam therapy in patients with previously untreated hepatocellular carcinoma. Cancer Sci 108: 497-503, 2017.

6. Tabrizian P, Jibara G, Shrager B, Schwartz M and Roayaie S: Recurrence of hepatocellular cancer after resection: Patterns, treatments, and prognosis. Ann Surg 261: 947-955, 2015.

7. Chan AC, Chan SC, Chok KS, Cheung TT, Chiu DW, Poon RT, Fan ST and Lo CM: Treatment strategy for recurrent hepatocellular carcinoma: Salvage transplantation, repeated resection, or radiofrequency ablation? Liver Transpl 19: 411-419, 2013.

8. Midorikawa Y, Takayama T,Higaki T, Nakayama H, Yamamoto M, Ariizumi S, Shimada K, Kokudo N, Tsuji S, Tsuchiya K, et al: Early hepatocellular carcinoma as a signaling lesion for subsequent malignancy. Jpn J Clin Oncol 46: 1102-1107, 2016.

9. Kudo M, Izumi N, Kokudo N, Matsui O, Sakamoto M, Nakashima O, Kojiro M and Makuuchi M; HCC Expert Panel Of Japan Society Of Hepatology: Management of hepatocellular carcinoma in Japan: Consensus-based clinical practice guidelines proposed by the Japan society of hepatology (JSH) 2010 updated version. Dig Dis 29: 339-364, 2011.

10. The Japan Society of Hepatology: Clinical practice guidelines for hepatocellular carcinoma (2013 version). https://www.jsh. or.jp/English/guidelines_en/Guidelines_for_hepatocellular_ carcinoma_2013.

11. Kim HJ, Lee KW, Kim YJ, Oh DY, Kim JH, Im SA and Lee JS: Chemotherapy-induced transient CEA and CA19-9 surges in patients with metastatic or recurrent gastric cancer. Acta Oncol 48: 385-390, 2009.

12. Mundle SD, Marathe AS and Chelladurai M: Transient therapy-related surge in serum tumor biomarkers: Characterizing behavior and postulating its biologic role. Crit Rev Oncol Hematol 86: 15-22, 2013.

13. Venniyoor A, Al Bahrani B and Rajan B: The dilemma of serum tumor marker (STM) flares. Gulf J Oncolog 1: 63-67, 2014.

14. Therasse P, Arbuck SG, Eisenhauer EA, Wanders J, Kaplan RS, Rubinstein L, Verweij J, Van Glabbeke M, van Oosterom AT, Christian MC and Gwyther SG: New guidelines to evaluate the response to treatment in solid tumors. European organization for research and treatment of cancer, national cancer institute of the United States, national cancer institute of canada. J Natl Cancer Inst 92: 205-216, 2000.

15. Iwata H, Ogino H, Hashimoto S, Yamada M, Shibata H, Yasui K, Toshito T, Omachi C, Tatekawa K, Manabe Y, et al: Spot scanning and passive scattering proton therapy: Relative biological effectiveness and oxygen enhancement ratio in cultured cells. Int J Radiat Oncol Biol Phys 95: 95-102, 2016.

16. Toshito T, Omachi C, Kibe Y, Sugai H, Hayashi K, Shibata H, Yasui K, Tanaka K, Yamamoto T, Yoshida A, et al: A proton therapy system in nagoya proton therapy center. Australas Phys Eng Sci Med 39: 645-654, 2016.

17. Nakajima K, Iwata H, Ogino H, Hattori Y, Hashimoto S, Nakanishi M, Toshito T, Umemoto Y, Iwatsuki S, Shibamoto Y and Mizoe JE: Acute toxicity of image-guided hypofractionated proton therapy for localized prostate cancer. Int J Clin Oncol 23: 353-360, 2018.

18. Hashimoto S, Shibamoto Y, Iwata H, Ogino H, Shibata H, Toshito T, Sugie C and Mizoe JE: Whole-pelvic radiotherapy with spot-scanning proton beams for uterine cervical cancer: A planning study. J Radiat Res 57: 524-532, 2016. 
19. Kanda Y: Investigation of the freely available easy-to-use software 'EZR' for medical statistics. Bone Marrow Transplant 48: 452-458, 2013

20. Song P, Cai Y, Tang H, Li C and Huang J: The clinical management of hepatocellular carcinoma worldwide: A concise review and comparison of current guidelines from 2001 to, 2017. Biosci Trends 11: 389-398, 2017.

21. Ueno M, Hayami S, Shigekawa Y, Kawai M, Hirono S, Okada K, Tamai H, Shingaki N, Mori Y, Ichinose M and Yamaue H: Prognostic impact of surgery and radiofrequency ablation on single nodular HCC $\leq 5 \mathrm{~cm}$ : Cohort study based on serum HCC markers. J Hepatol 63: 1352-1359, 2015.

22. Park SJ, Jang JY, Jeong SW, Cho YK, Lee SH, Kim SG, Cha SW, Kim YS, Cho YD, Kim HS, et al: Usefulness of AFP, AFP-L3, and PIVKA-II, and their combinations in diagnosing hepatocellular carcinoma. Medicine (Baltimore) 96: e5811, 2017.

23. Yu R, Tan Z, Xiang X, Dan Y and Deng G: Effectiveness of PIVKA-II in the detection of hepatocellular carcinoma based on real-world clinical data. BMC Cancer 17: 608, 2017.

24. Sherman M: Recurrence of hepatocellular carcinoma. N Engl J Med 359: 2045-2047, 2008

25. Bae JS, Park SJ, Park KB, Paik SY, Ryu JK, Choi CK and Hwang TJ: Acute exacerbation of hepatitis in liver cirrhosis with very high levels of alpha-fetoprotein but no occurrence of hepatocellular carcinoma. Korean J Intern Med 20: 80-85, 2005 .
26. Xing H, Yan C, Cheng L, Wang N, Dai S, Yuan J, Lu W, Wang Z, Han J, Zheng Y and Yang T: Clinical application of protein induced by vitamin $\mathrm{K}$ antagonist-II as a biomarker in hepatocellular carcinoma. Tumour Biol: Oct 13, 2016 (Epub ahead of print).

27. Takamatsu S, Yamamoto K, Maeda Y, Kawamura M, Shibata S, Sato Y, Terashima K, Shimizu Y, Tameshige Y, Sasaki M, et al: Evaluation of focal liver reaction after proton beam therapy for hepatocellular carcinoma examined using Gd-EOB-DTPA enhanced hepatic magnetic resonance imaging. PLoS One 11: e0167155, 2016.

28. Imada $H$, Kato $H$, Yasuda $S$, Yamada $S$, Yanagi $T$, Hara $R$, Kishimoto R, Kandatsu S, Minohara S, Mizoe JE, et al: Compensatory enlargement of the liver after treatment of hepatocellular carcinoma with carbon ion radiotherapy-relation to prognosis and liver function. Radiother Oncol 96: 236-242, 2010.

29. Arora A and Kumar A: Treatment response evaluation and follow-up in hepatocellular carcinoma. J Clin Exp Hepatol 4 (Suppl 3): S126-S129, 2014.

30. Brook OR, Thornton E, Mendiratta-Lala M, Mahadevan A, Raptopoulos V, Brook A, Najarian R, Sheiman R and Siewert B: $\mathrm{CT}$ imaging findings after stereotactic radiotherapy for liver tumors. Gastroenterol Res Pract 2015: 126245, 2015.

31. Lencioni R and Llovet JM: Modified RECIST (mRECIST) assessment for hepatocellular carcinoma. Semin Liver Dis 30: 52-60, 2010

32. Kudo M, Ueshima K, Kubo S, Sakamoto M, Tanaka M, Ikai I, Furuse J, Murakami T, Kadoya M and Kokudo N; Liver Cancer Study Group of Japan: Response evaluation criteria in cancer of the liver (RECICL) (2015 revised version). Hepatol Res 46: 3-9, 2016. 